\title{
Magnetic Microwires With Field-Induced Helical Anisotropy for Coil-Less Fluxgate
}

\author{
M. Butta ${ }^{1}$, P. Ripka ${ }^{1}$, G. Infante ${ }^{2}$, G. A. Badini-Confalonieri ${ }^{2}$, and M. Vázquez ${ }^{2}$ \\ ${ }^{1}$ Faculty of Electrical Engineering, Czech Technical University, 16627 Prague, Czech Republic \\ ${ }^{2}$ Institute for Materials Science of Madrid, CSIC, 28049 Madrid, Spain
}

\begin{abstract}
We present a new method for production of magnetic microwire with helical anisotropy. Coil-less fluxgate sensors are generally composed of a bimetallic wire excited by an alternating current; in order for the wire to work in coil-less fluxgate mode, the magnetic layer of the wire needs to have helical anisotropy. So far, we have achieved such anisotropy by mechanically twisting the wire. However, this method has some disadvantages for practical applications, mainly regarding the sensor stability. We propose a method that provides helical anisotropy by applying a helical field during the electrodeposition: this is achieved by the superposition of a longitudinal field generated by a Helmholtz coil and a circumferential field produced by a direct current flowing through the core of the wire during electrodeposition.
\end{abstract}

Index Terms-Anisotropy, coil-less, fluxgate, field induced, helical, microwires, orthogonal.

\section{INTRODUCTION}

C OIL-LESS fluxgates are a special type of orthogonal fluxgate. They are composed of a single magnetic wire, excited by the current flowing into the wire itself; the output voltage is picked up directly from the terminations of the wire, without the need of a pickup coil. Therefore, they have a simpler and smaller structure compared to traditional fluxgate sensors.

In order for a magnetic microwire to work in coil-less fluxgate mode, we need helical anisotropy. So far, mechanical deformation has been used to induce helical anisotropy: by applying torsion on the terminations of the wire, we are able to control the anisotropy angle. In [1], we have shown that the sensitivity of the output voltage depends on the angle of the helical anisotropy.

Despite the fact that anisotropy induced by twisting proved to make the magnetic wire work as desired, it is not suitable for practical applications. Indeed, the need to apply a constant torque to the wire complicates the structure of the sensor; it is not easy to keep a constant tension. Any change of torque results in a change of sensitivity, and therefore of the output voltage. In this way, the sensor's output strongly depends on temperature, because any change of temperature could modify the geometry of the supports which produce the torque.

In this paper, we will show how we produced magnetic microwires with built-in helical anisotropy, which do not need any mechanical stress to work as coil-less fluxgate.

\section{BI-METALLIC WIRE}

Bi-metallic wires have proven to be a good solution for core of orthogonal fluxgate, including coil-less fluxgate. They are composed of a copper core, where the current is injected, covered by a layer of magnetic material. The current flowing into the copper core generates a circumferential magnetic field which saturates the surrounding magnetic layer. The external field to be measured is applied in longitudinal direction and it modifies the direction of the magnetization.

Magnetic microwires with an insulation layer between the copper core and the magnetic layer have been proposed: if there is no conductive path between the copper core and the magnetic layer, we can keep the current flowing only into the copper region, without concentration of the current to the magnetic layer at increasing frequency. This has been proven to help reduce the current amplitude to achieve full saturation of the microwire [2].

This kind of wire is obtained using a glass-coated copper wire as a base. The terminations are properly shielded by plastic film, and a free nm layer of gold is sputtered on the surface of the glass. Due to the small diameter of the wire, it is rather easy to obtain a uniform sputtered layer all over its circumference, despite the fact that only one side of the wire is facing the gold target during the sputtering (and the wire is not moving).

A Permalloy layer is then applied on the glass layer by means of an electrodeposition process. The current necessary to determine electrodeposition is injected on the glass layer on the termination which is kept out of the electrodeposition bath. During the electrodeposition the copper core is not involved at all: the termination dipped into the bath is at the same potential as of the bath, but the termination kept out of the bath is not connected to any potential: therefore, the copper core does not carry any current.

As already mentioned such wires proved to have low saturation current, when compared to other bi-metallic CopperPermalloy wires without insulation layer (in a usual process the Permalloy is electroplated directly on the copper wire). However, they still required mechanical torsion to work as coil-less fluxgate. In fact, we could not observe any spontaneous coil-less working mode in the wire without the applied torque.

\section{FIELD-INDUCED HeliCAl ANISOTROPY}

Manuscript received December 16, 2009; revised February 16, 2010 accepted March 09, 2010. Current version published June 23, 2010. Corresponding author: M. Butta (e-mail: buttam1@ fel.cvut.cz).

Color versions of one or more of the figures in this paper are available online at http://ieeexplore.ieee.org.

Digital Object Identifier 10.1109/TMAG.2010.2045885
In order to produce helical anisotropy in the electrodeposited magnetic layer, we must apply a helical field strong enough during the electrodeposition process. 


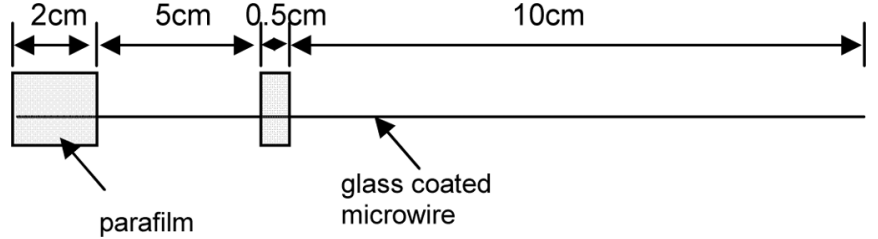

Fig. 1. Preliminary preparation of the glass-coated copper wire for sputtering.

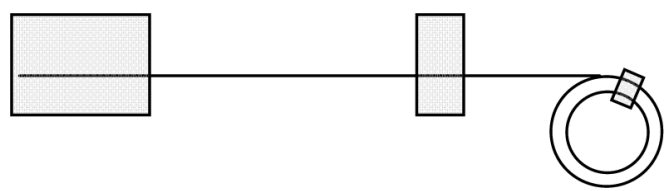

Fig. 2. Preliminary preparation of the glass-coated copper wire for sputtering.

It has been reported that a moderate magnetic field during electroplating can modify the magnetic properties of $\mathrm{NiFe} / \mathrm{Cu}$ composite wires [3] and also for other alloys/geometries [4], [5].

Moreover, it has been reported that magnetic field has a beneficial effect on compactness and deposit uniformity, growth orientation, etc. [4] (cited 46 times), [5], [6]. But in most of these works a much stronger field (up to several T) is employed.

In order to generate a helical field, first we tried to simply apply a longitudinal field along the wire by means of a Helmholtz coil. We supposed that the combination of such longitudinal field with the field produced by the electrodeposition current flowing into the gold layer along the wire would have resulted in a helical field. In fact, no helical anisotropy was observed.

This is clearly due to the fact that the electrodeposition current is too low to generate a circumferential field strong enough to give anisotropy.

For instance, if we consider a $1 \mathrm{~mA}$ electrodeposition current (the highest value ever used to produce such wires) and a $50 \mathrm{~mm}$ maximum diameter wire, we obtain $6 \mathrm{~A} / \mathrm{m}$ circumferential field, which is basically negligible. A much stronger circumferential field must be applied, together with the longitudinal field, in order to achieve a helical anisotropy.

We have therefore modified the process for the production of the magnetic microwires as follows.

First, we prepare a $\sim 17-\mathrm{cm}$-long glass-coated copper microwire and we apply a film on a termination and a second short film after $5 \mathrm{~cm}$ (Fig. 1).

Then, we wound the 10-cm-long side of the wire on a plastic ring and we apply another short strip of plastic film to keep the wire on the ring (Fig. 2).

Then, we put the wire in the sputtering chamber, fixing the plastic films on the sample holders (Fig. 3).

After the sputtering process, the central part of the wire will be completely covered by the gold layer. In fact, other parts of the wire will be covered by gold, because they are not shielded by the film, as shown in Fig. 4. However we consider only the first part of the wire covered by gold for electrodeposition: the current which carries the Permalloy on the gold stops flowing at the first interruption of the gold layer; therefore, we do not care

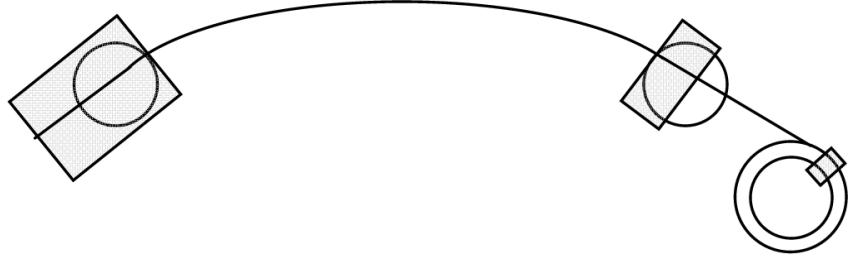

Fig. 3. Arrangement of the microwire on sample holders for electrodeposition. The central part of the wire is sputtered, and will be used for electrodeposition.

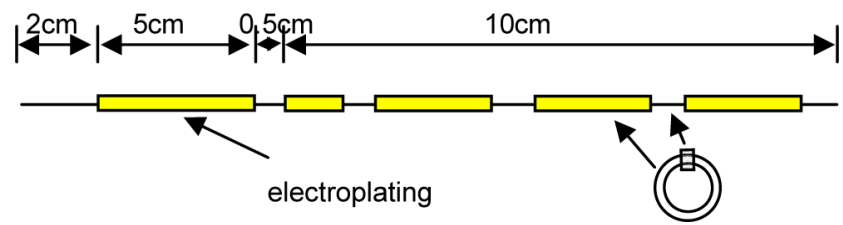

Fig. 4. Microwire after sputtering. The plastic film breaks the continuity of the gold layer.

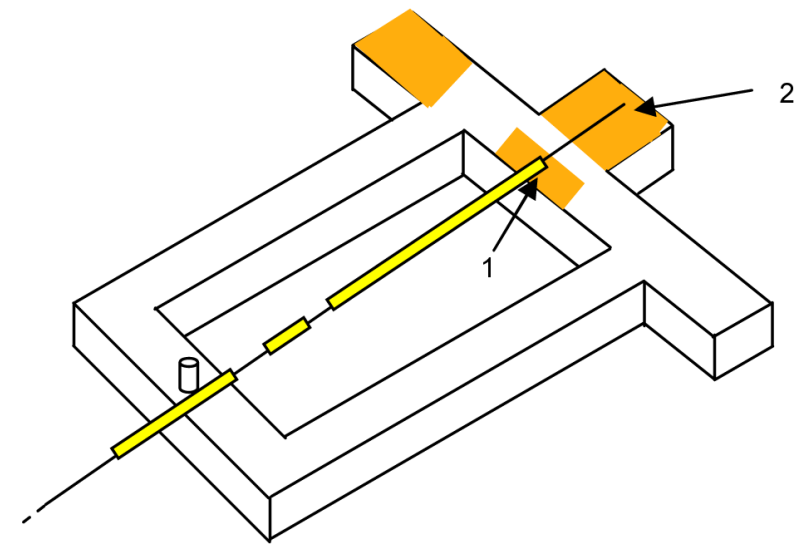

Fig. 5. Connection of the gold layer (1) and copper core termination (2) to the sample holder contacts.

about other sections of the wire covered by gold, because they will not be active for electrodeposition.

Finally, the sputtered wire is fixed on a special sample holder for electrodeposition as shown in Fig. 5.

The sample holder has three copper contacts: the one marked as " 1 " is connected to the terminal part of the gold sputtered layer, and it will carry the current for electrodeposition. The contact marked as " 2 " is connected to the copper core, after removal of a glass coating on the termination of the wire. Then the floating side of the wire is turned up and fixed at its termination at contact marked " 3 ", in Fig. 6. As for the contact " 2 " also contact " 3 " is connected to the copper core, after removing the glass coating. All three contacts are fixed using conducting silver paint. When fixing the contact 3 , we take care not to apply any mechanical stress to the wire.

The sample holder is dipped into the electrodeposition bath, keeping the three contacts out of the bath.

The bath is located in the middle of a Helmholtz coil, which produces a magnetic field along the axes of the wire (Fig. 7). A first generator (on the left) is connected on the bath and to the gold layer, through the mentioned contact " 1 ", and it carries the electrodeposition current. Another generator (on the right) is connected on the two mentioned contacts " 2 " and " 3 ", that is on 


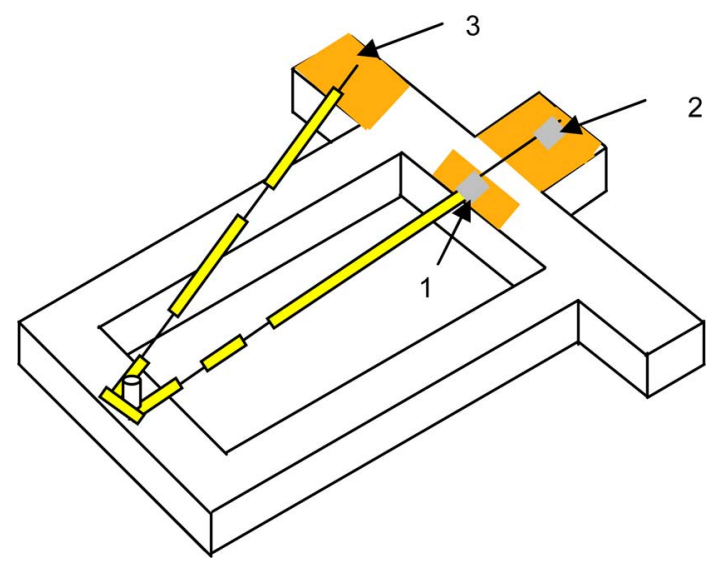

Fig. 6. Connection of the second termination of the copper core (3) to the contact of the sample holder. During this connection, we take care not to apply any tension to the wire.

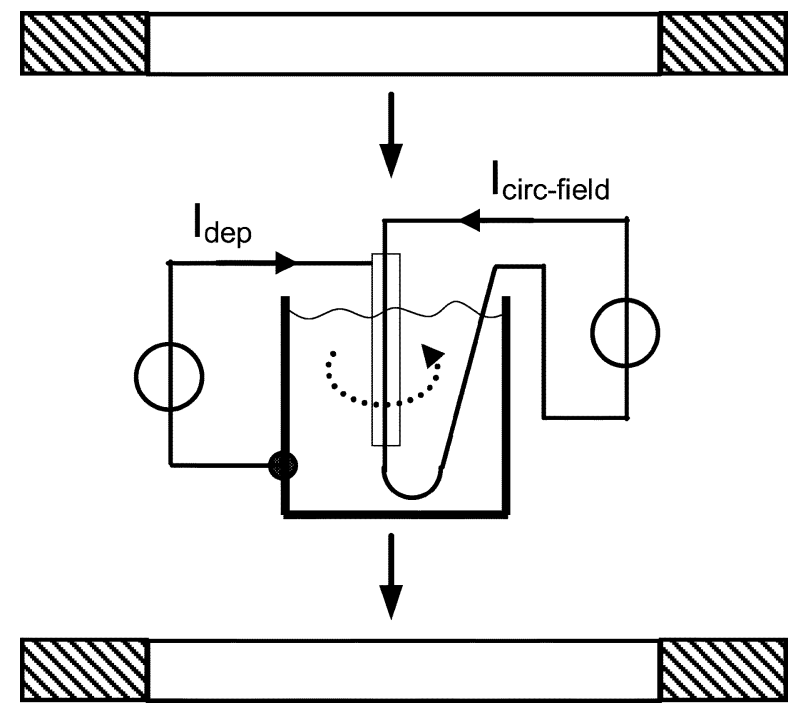

Fig. 7. Electrodeposition setup.

the terminations of the copper core. Due to galvanic insulation provided by the glass coating, and due to the fact that all contacts are outside the bath, the two circuits are absolutely independent.

We generate a $600 \mathrm{~A} / \mathrm{m}$ longitudinal field using the Helmholtz coil; simultaneously we inject a $150 \mathrm{~mA}$ dc current into the copper core to generate a $\sim 450 \mathrm{~A} / \mathrm{m}$ circumferential field. We have verified that the copper core can tolerate even three times higher dc current without any problem.

\section{RESULTS}

By using the method previously described, we have produced a magnetic microwire with built-in helical anisotropy, which works in coil-less mode, without any need of mechanical stress.

Fig. 8 shows the circumferential $B-H$ loop of one of the wires produced by this method and the application of $\pm 20 \mathrm{~A} / \mathrm{m}$. The $B-H$ loop shifts like it is supposed to do when it works in coil-less fluxgate mode. If helical anisotropy was not present, the $B-H$ loop would not shift, but it would be symmetrically deformed by reduced amplitude.

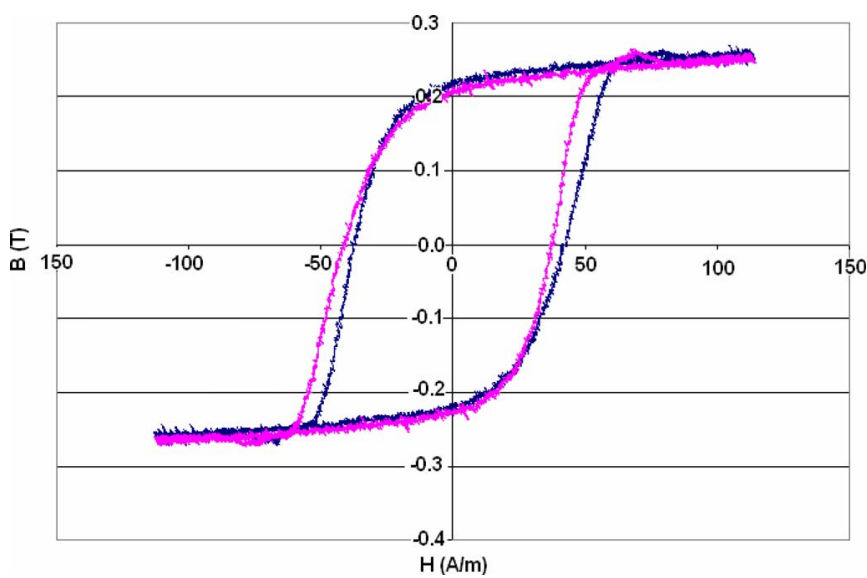

Fig. 8. Circumferencial $B-H$ loop of produced microwire, under the effect of $\pm 20 \mathrm{~A} / \mathrm{m}$. The loop shifts due to helical anisotropy.

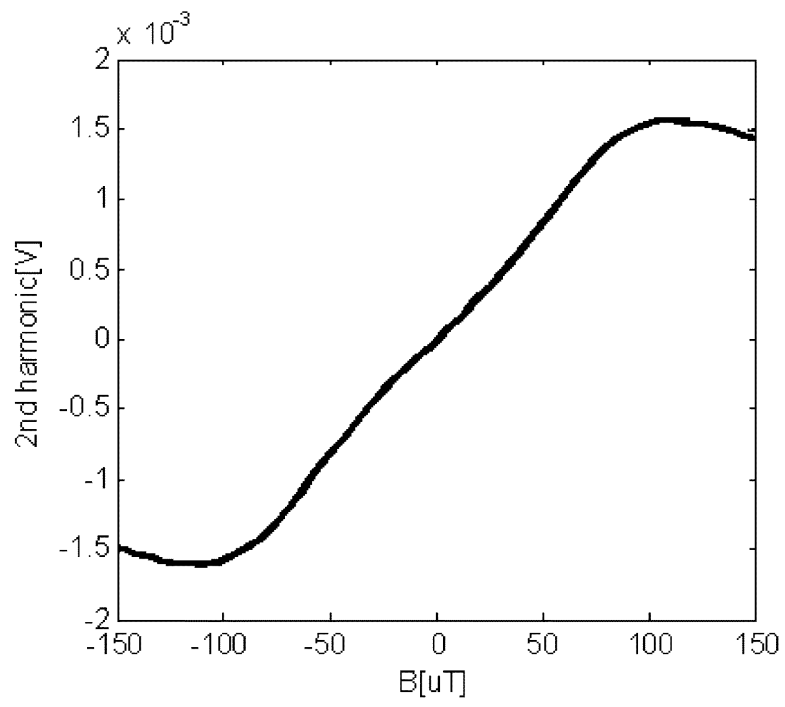

Fig. 9. Second harmonic response of sinewave excited wire $(30 \mathrm{~mA}$ peak, $10 \mathrm{kHz}$ ), produced with built-in helical anisotropy and working in coil-less mode.

We have finally tested the output characteristic of the wires by using simple sinewave excitation current $(30 \mathrm{~mA}$ peak, $10 \mathrm{kHz})$, and second harmonic extraction.

Fig. 9 shows the dependence of second harmonic on the external applied field. We can observe that there is a linear region around $\pm 40 \mu \mathrm{T}$ : this region corresponds to the mentioned behavior of the $B-H$ loop shifting to the left or to the right, due to external field and helical anisotropy. For higher field amplitude we observe another linear region with slightly higher sensitivity: in this case the output is given by a deformation of the $B-H$ loop and not just by its well known shifting mechanism. We will neglect this phenomenon and we will consider only the central linear part of the characteristic.

We have produced wires changing the amplitude of both longitudinal and circumferential field, in order to change the anisotropy angle. Our aim was to show a dependence of the sensitivity on the so changed anisotropy angle. Unfortunately, we could not correctly compare the sensitivity of the different wires where the only changing parameter was the anisotropy 
angle. At the present stage of the technology, the thickness of the wire is not always constant and the quality of the bath is changing.

\section{FURTHER IMPROVEMENTS}

During the production of magnetic microwires with built-in helical anisotropy, we have observed some particular phenomenon. We have tried to produce wires with longer electrodeposited region, in order to increase the sensitivity. In this case, we observed regions with different anisotropy angle. Indeed, we had a $B-H$ loop shifting in two stages. This is due to the fact that the field produced by the Helmholtz coil is uniform only for a limited region in the middle of the coil.

If the wire is too long, it turns out that different portions of the wire are exposed to different amplitude of longitudinal field. Therefore, we had to limit the length of the Permalloy layer to a maximum of $\sim 2 \div 3 \mathrm{~cm}$ (the total $5 \mathrm{~cm}$ length of sputtered gold includes also the part of the gold out of the bath which is not electrodeposited).

We believe that an improvement could be achieved by using a bigger Helmholtz coil, which can provide a bigger area of uniform field.

Another source of the problem is the uniformity of the circumferential field. As the electrodeposited layer grows, the new section added to the layer will be under a decreasing field. The circumferential field decreases as $\sim 1 / \mathrm{r}$ (where $\mathrm{r}$ is the distance from the center of the wire); therefore, the difference between the circumferential field when we start and when we finish the electrodeposition could not be negligible. In order to keep the circumferential field constant during the whole electrodeposition process, and therefore to obtain a constant anisotropy angle over the whole thickness of the layer, we should increase the current flowing the copper core, in order to compensate the reduction of field due to the distance.

\section{CONCLUSION}

In this paper, we have proposed a novel method to produce magnetic microwire with built-in helical anisotropy, without any mechanical stress. These wires are suitable for cores of coil-less fluxgates.

We have also proposed an idea how to further improve the process of electrodeposition under the effect of helical field, in order to obtain a constant anisotropy angle.

\section{ACKNOWLEDGMENT}

This paper was presented at the 19th Soft Magnetic Materials Conference. See IEEE TRANSACTIONS ON MAGNETICS, vol. 46, no. 2, February 2010.

\section{REFERENCES}

[1] M. Butta, P. Ripka, S. Atalay, F. E. Atalay, and X. P. Li, "Fluxgate effect in twisted magnetic wire," J. Magn. Magn. Mater., doi:10.1016/j. jmmm.2008.04.176.

[2] M. Butta, P. Ripka, G. Infante, G. A. Badini-Confalonieri, and M. Vázquez, "Bi-metallic magnetic wire with insulating layer as core for orthogonal fluxgate," IEEE Trans. Magn., vol. 45, no. 10, pp. 4443-4446, Oct. 2009

[3] X. P. Li et al., "Effect of magnetic field on the magnetic properties of electroplated $\mathrm{NiFe} / \mathrm{Cu}$ composite wires," J. Appl. Phys., vol. 94, p. 6655,2003

[4] A. G. Muñoz et al., "Magneto impedance of electroplated NiFeMo-Cu microwires for magnetic sensors," J. Phys. D: Appl. Phys., vol. 40, p. 5013, 2007.

[5] V. Georgescu et al., "Magnetic field effects on surface morphology and magnetic properties of Co-Ni-P films prepared by electrodeposition,"' Surface Sci., vol. 600, p. 4195, 2006.

[6] T. Z. Fahidy, "Characteristics of surfaces produced via magnetoelectrolytic deposition,” Prog. Mat. Sci., vol. 68, p. 155, 2001.

[7] A. Ispaset al, "Influence of a magnetic field on the electrodeposition of nickel-iron alloys," Electrochim. Acta, vol. 52, p. 2785, 2007.

[8] J. A. Koza et al., "The effect of magnetic field on the electrodeposition of CoFe alloys," J. Magn. Magn. Mater., vol. 321, p. 2265, 2009. 